\title{
A RECENT DEVELOPMENT IN NISAN SYSTEM
}

\section{Asano, Choichiro}

Research Institute of Fundamental Information Science, Kyushu University | Department of Information Systems, Interdisciplinary Graduate School of Engineering Science, Kyushu University

\section{Watanabe, Michiko}

Research Institute of Fundamental Information Science, Kyushu University | Department of Information Systems, Interdisciplinary Graduate School of Engineering Science, Kyushu University

Hosokawa, Koj i

Department of Information Systems, Interdisciplinary Graduate School of Engineering Science, Kyushu University

Kurihara, Koji

Department of Information Systems, Interdisciplinary Graduate School of Engineering Science, Kyushu University

https://doi.org/10.5109/13338

出版情報: Bulletin of informatics and cybernetics. 20 (1/2), pp.79-96, 1982-03. Research Association of Statistical Sciences バージョン :

権利関係 : 


\title{
A RECENT DEVELOPMENT IN NISAN SYSTEM
}

\author{
By
}

\author{
Chooichiro Asano ${ }^{* * *}$, Michiko Watanabe***, Koji HosokaWa** \\ and
}

Koji KURIHARA**

(Received November 26, 1981)

\begin{abstract}
NISAN system has been developed by a group of Japanese statisticians, since 1978. The package is widely applicable in statistical data analyses, confirmatory and exploratory, with newly developed methods. The system is useful not only for the beginners in data analysis, but also for the senior statisticians with analytical insight, i.e. choosing adequate procedure of statistical analysis and model-building.

In the presest paper, some features in NISAN system recently developed are presented, especially concentrating on both functions of new monitor and knowledge-commands. These two functions are mainly for statistical researchers. The former is easy to cooperate and to accumulate further commands developed with respect to datahandling and analytical methods by NISAN research members, working in the respective fields of statistics and locating in several parts of Japan. The latter is to investigate and to evaluate properties of statistical methodologies by simulations in some varieties of circumstances given, and jointly may be to recognize the mathematical is odel previously unspecified for data.
\end{abstract}

\section{Introduction}

NISAN system is a Ntw interactive Statıstical ANalysis program package, which consists ma nly of the following five modules: NISAN-MONITOR module for control, STAT module for sta istical methods in confirmatory and exploratory analysis of data, DATA module for data basti, HELP module for various inquiries with three levels, and DOC module for documentation. The conversational terminals are cathode ray tubes, teletypes or graphic terminais. where all the outputs in NISAN system are designed on at most eighty characters per a line.

In this way, NISAN system has been developed concretely not only on ordinarily important commands for methods of statistical data analysis, but also on some commands for statistical data-handling, e.g. stratification, graphic representation, quantification and

* Research Institute of Fundamental Information Science, Kyushu University 33, Fukuoka 812.

** Department of Information Systems, Interdisciplinary Graduate School of Engineering Science, Kyushu University 39, Fukuoka 812. 
so on. Thus a relatively simple users' guide was made on this February as a trial, and the booklet obtained good evaluation for statisticians without regard to their experience of computer work. Therefore more commands of analytical methods and statistical data-handling are now reinforced.

On the other hand, two emphasized features, that is, a new NISAN monitor with effective functions and some knowledge-commands for statistical inference are recently studied and developed with the ordinary method-commands.

\section{New NISAN-monitor}

Generally speaking, the functions of monitor are very important for efficiency of the whole system of statistical program package. Regarding the program construction of NISAN system, the main functions of NISAN-monitor are as follows: interpretation of input commands, allocation of memories, allocation of files, control of several peripheral devices, terminals and programs.

In NISAN system, the monitor is fully written by the standard FORTRAN IV language, although only LOGON and BREAK functions depend on TSS. This assures every university computer of the portability of NISAN system, that is to say, NISAN system is independent from the kinds of computer machines, being available FORTRAN language.

The monitor interprets naturally regular commands and operands. In them, some conventions are particularly provided for users' convenience. For example, variables $\mathrm{X} 1: \mathrm{X} 5$ means all variables between $\mathrm{X} 1$ and $\mathrm{X} 5$ in NISAN file, variables $\mathrm{X}(1: 5)$ means $\mathrm{X} 1, \mathrm{X} 2, \mathrm{X} 3, \mathrm{X} 4$ and $\mathrm{X} 5$,

values $\quad 1: 5$ means values between 1 and 5 , i.e. $[1,5]$,

and so on. Also several conventions, depending on the command, are given as shown in the following example;

levels $\mathrm{L}((\mathrm{X} 1 \mathrm{1}$ (2 7 8) (3 4)) means making three levels of variable X1, where the respective levels are composed of a value 1 , a set of values $2,7,8$ and a set of values 3,4 , as elements.

For the convenience of successive replication of analysis, changing variables is considered simultaneously by arranging related variables, as shown in the following example,

SCAT(X1 X2:X5) means four scatter diagrams of X2, X3, X4 and X5 on X1, respectively.

Regarding operands, many varieties are prepared to obtain several features of outputs corresponding to users' requests. In case of omission for operands, either a prompt inquiry is presented or a standard value involved is given, depending on the semantics of the operands. In the monitor, the interactive functions are provided.

In view of statistical analysis, it makes clear in NISAN system for us to use statistical terminologies, defined by mathematical statistics, as the names of commands and operands. For example,

POP means POPulation(s).

TESTIM means statistical TESTs and ESTIMation.

REF means RElative EFficiency.

DEFTP means DEFinition of Test-Process. 
The NISAN standard file is applied as a self-descriptive systematized file composed of both parts of directory and data, since there exist big varieties on types and formats in every file of users. The logical structure of data part is a set of record, being called cases. The file has parent file name, and each case consists of a set of items refered by variable names. Group names of variables are also assigned as key names, and in case of continuous type of variables, three data ranges are given as normal, warning and something unusual.

It is one of the most emphasized functions of NISAN monitor for the research members to construct easily new commands with operands on the basis of their FORTRAN programs. The arrangement is fill several definitions for commands and operands in a printed form, and to set initial values for arguments. Thus the setup is completed by a certain simple operations on TSS. As the manual, a thin booklet Commands Construction Manual for NISAN Research Members [3] is prepared to be easily to understand for statisticians, even if they are not so familiar with computer operation.

In view of further development of NISAN system, this function of the monitor is very useful, since the research members of the organization of NISAN system are widely located at several universities and institutions in Japan, and the respective members, working in various statistical fields, are able to integrate easily their authorized programs of analytical methodologies to NISAN system.

\section{Analytical method-commands}

It is natural in statistical program packages to have many method-commands to analyze actual data in data file. In NISAN system, analytical programs in STAT module are originally developed and are integrated under TSS operation. Although the whole method-commands involved are still not so many and such commands are soon to be added, a tentative users' guide of 43 pages was prepared as a trial in this February. Since the manner of writing and form was simple and customary, the booklet had a good reputation to apply easily those commands and operands. In order to show some illustrations, several commands are quoted from the booklet in the following manner.

The contents of the respective commands are composed of (0) Name of command, (1) Input form, (2) Functions, (3) Explanation of operands, (4) Pagination of illustrations of output.

\section{Knowledge-commands}

\subsection{The principle}

NISAN system is widely available for both statistical situations, confirmatory analysis and exploratory analysis, and is not only for senior statisticians to obtain analytical wisdom on mathematical model-building for the essence of urgent phenomenon, but also for the beginners of statistical data analysis to learn analytical methods and properties and to train their experience of data analysis. For these purposes in NISAN system, separating from analytical method-commands, so-called knowledge-commands have been developed to investigate or to learn about methodologies of statistical in- 


\section{Command MEAN2}

Significance test between two sample mean vectors in multivariate normal population with unknown variancecavariance.

MEAN2 [FI (file name) $]^{*}$

[VAR (variable name list) ]*

POP (identification variable name value 1 value 2 )

[ALPHA (significance level)]

\section{Command HIST}

Output of histograms for respective variables.

$\begin{aligned} \text { HIST } & {[\text { FI (file name) }]^{*} } \\ & {[\text { VAR (variable name list) }]^{*} } \\ & {[\text { MINIMUM (minimum value) }] } \\ & {[\text { MAXIMUM (maximum value) }] } \\ & {[\text { SIZE (number of intervals) }] } \\ & {[\underline{\text { DENSITY (frequencies for a }}} \\ \text { height scale unit) }] & {[\text { CUMULATE }] \text { [NUMERAL }] }\end{aligned}$

12. Command SCAT

Scatter diagrams of the second and the latter variables on the first variable respectively.

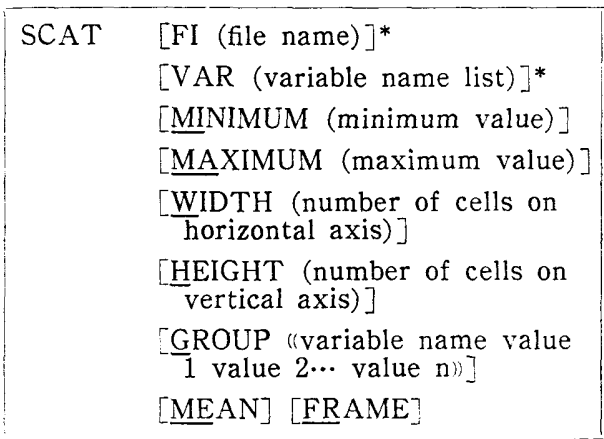

\section{Command STREG}

Stepwise forward or backward regression analysis with a constant term.

\begin{tabular}{|c|c|}
\hline STREG & 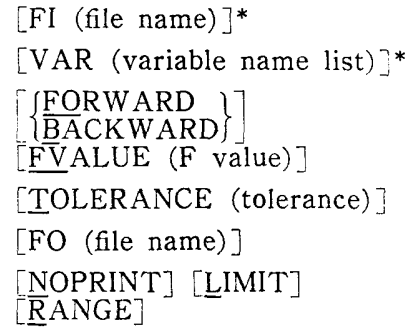 \\
\hline
\end{tabular}

\section{Command LEVEL}

Level-making of each variable before cross-table analysis, analysis of variance, and so on.

LEVEL FI (file name)

L ("variable name level 1 level $2 \cdots$ level $\mathrm{n}) \cdots$ )

\section{Command CROSS}

Making two-way cross tables.

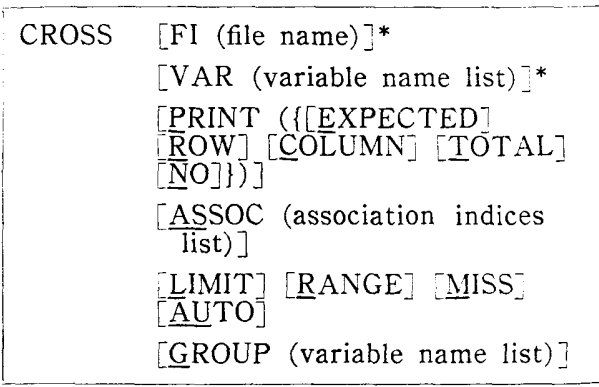

20. Command MNT

Multivariate normality test, including 1. skewness 2. kurtosis 3. Geary's test 4. Shapiro-Wilk 5. Shapiro-Francia 6. D'Agostino-Pearson

$\begin{aligned} \text { MNT } & {[\mathrm{Fl} \text { (file name) }]^{*} } \\ & {[\mathrm{VAR} \text { (variable name list) }]^{*} }\end{aligned}$

\section{Command CLUST}

Cluster analysis based on distance or similarlity.

\begin{tabular}{|c|c|}
\hline CLUST & 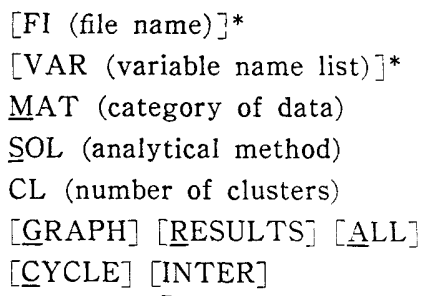 \\
\hline
\end{tabular}

Note

(1) [ ] means that operand and item involved can be omitted.

(2) []$^{*}$ means that operand and item involved are not necessary when they are fixed previously by LOCK command.

(3) \{ $\}$ means that only one operand must be selected from the plural operands shown.

(4) Underline of operand means the shorten ing form. 
ference, including the single method and the serial process of methods for inference. In such simple situations, facing the statistical data analysis, one likes to obtain statistical knowledge about the properties of applied methods on the accuracies and precisions for estimates, the distributions or conditional distributions of test statistics, the powers of tests, and furthermore the relative efficiencies or the asymptotic relative efficiencies between two arbitrary tests in case of selecting statistical tests for several alternative hypotheses in varieties of circumstances to be considered. On these accounts, commands DIST, POWER, REF and AREF are available.

From the viewpoint of the process of statistical inference, generally speaking, in order to specify an adequate mathematical model for observations or to investigate the feature pattern of data, statisticians apply more or less their systematic processes, composed of several methods, to their data basing upon their logics of inference.

As a simple example of inference process, called a kind of pooling methodology by Bancroft et al., let us consider an estimation problem for two observation means with a common but unknown variance. Then it may be natural that when the distinction between two observation means is not significant by $t$-test, two sets of observations may be pooled and the average is given as the best estimates of both population means, because of acceptance of the null hypothesis that two population means are equal. On the other hand, if the distinction is significant, the respective observation means may be given as the respective best estimates of population means. More details are discussed in section 4.3. Ordinary processes of statistical inference may be composed of more tests, and the mathematical formulations are extremely complicated with multiple integrations on complex domain.

Even if the inference process is multiple-stage, NISAN system is able to give very quickly numerical evaluation of the properties with enough precision about inference on the basis of simulating the inference process by NISAN random numbers or by random number generation implemented and arbitrarily transformed as in Table B, keeping away from awfully complicated mathematical formulations and these numerical computations.

Such inference processes are also applied frequently in case of nonparametric inference, since the structure of observations is too complicated to know what kinds of nonparametric tests are suitable to the data feature, which may be observed from a composite population. Since every test itself has a specific aspect without a consistence in the test-statistic, and actually several nonparametric tests are tried. Then some tests indicate significant but another indicate nonsignificant. Therefore, in cases of exploratory analysis or in the situations of imcompletely specified model of data, it may be dissatisfied for us to apply merely a statistical test. It may be needed ordinarily to take a test process.

Thus at any rate, the performances and properties of such inference processes have to be theoretically evaluated and, if any, the optimality improvement should be considered as statistician's knowledge. For instance, the overall power of a test-process, the relative efficiency between arbitrary two test-processes, the accuracy and precision of estimate due to preliminary test-process should be studied.

These knowledges may be also able to prevent misapplication of statistical methods 
and of statistical program packages, and jointly to recognize the mathematical model of data.

For these purposes, knowledge-commands DEFTP, CONDIST, TPATTN, POWER and TESTIM are prepared in NISAN system. In the following section, more details of the commands are introduced.

\subsection{Formation of each command}

The manner of writing and form is, of course, similar with the method-commands but a little complicated in users' guide. In order to show concisely their functions, operands and varieties, we quote the expression of these commands in its entirety, as below.

\section{(i) Command DIST}

\begin{tabular}{|c|c|}
\hline DIST &  \\
\hline
\end{tabular}

[Function]

To obtain the distribution of a test statistic in a certain environment given.

[Operands]

TEST …A test name shown in Table A. POP $\quad \cdots$ The number of populations.

SIZE $\cdots$ The respective sizes of samples obtained from the populations.

TYPE $\cdots$ A name of type of random numbers shown in Table B. In case of omittion of key-in, NOR is applied.

HIST $\cdots$ A histogram of test statistic is shown by the simulation. In case of omittion of PERC. and RERT-commands, the histogram of test statistic is shown, even if HIST command is omitted.

SIM $\cdots$ The size of simulation in a cycle. In case of omittion, the size is five thousands.

PARA ‥Parameters of the random numbers defined by TYPE.
UP $\quad$... Output of the upper probability level, or the upper percentile of test statistic.

LW $\quad$.. Output of the lower probability level, or the lower percentile of test statistic.

BO $\quad$... Output of either sides of the probability level, or of the both percentiles.

PERC …utput of the probability level for the percentile given.

PERT …utput of the percentile for the probability level given.

\section{(ii) Command POWER}

POWER TEST (test name)

POP (number of populations)

SIZE (sample 1 sample $2 \cdots$ )

[TYPE (type of R.N.)]

[ALPHA (probability level)]

[SIM (size of simulation)]

[PARA (parameters of R. N.)]

[SIG (pattern of sig.)]

[Function]

To obtain the power of test given.

[Operands]

TEST ...The name of a test shown in Table A, or the name of a test process defined by DEFTP command.

SIG … Only if a test process si applied in TEST operand, SIG operand is needed. The manner is the same as DEFTP command.

The other operands are quite the same as 
those in DIST command.

\section{(iii) Command REF}

\begin{aligned} \hline REF & $\left.\frac{\text { TEST (test name } 1 \text { test name }}{2}\right) \\ &$ POP (number of populations) \\ & SIZE (sample 1 sample $2 \cdots) \\ & {[$ TYPE (type of R. N.) $} \\ & {[$ ALPHA (probability level) $] } \\ & {[$ SIM (size of simulation)] $} \\ & {[$ PARA (parameters of R.N.)] $}\end{aligned}$

[Function]

To obtain the relative efficiency of test 2 to test 1 in a certain environment given.

\section{[Operands]}

TEST …The name of tests shown in Table A, or the name of test process(es) defined by DEFTP command.

The other operands are quite the same as those in DIST command.

\section{(iv) Command AREF}

\begin{tabular}{|ll}
\hline AREF & $\left.\frac{\text { TEST (test name } 1 \text { test name }}{2}\right)$ \\
& POP (number of populations) \\
& SIZE (sample 1 and $2 \cdots)$ \\
& {$[$ TYPE (type of R. N.) $]$} \\
& {$[$ ALPHA (probability level) $]$} \\
& {$[$ SIM (size of simulation) $]$} \\
\hline
\end{tabular}

[Function]

To obtain the asymptotic relative efficiency of test 2 to test 1 in a certain environment given, where test 1 is to be the best test in the Eeden's sense.

[Operands]

Operands involved are quite the same as those in REF command.

\section{(v) Command DEFTP}

\begin{tabular}{rl|}
\hline DEFTP & NAME (test process name) \\
& TEST (test 1 …test $n+1)$ \\
& SIG (pattern of sig.) \\
& $[$ ALPHA (lev. $1 \cdots$ lev. $n)]$ \\
\hline
\end{tabular}

\section{[Function]}

To define a test process (test-series), where individual tests are in Table A.

[Operands]
NAME $\cdots$ The name of a test process to be defined.

TEST ...The names of tests applied in a test process.

ALPHA ...Significance levels for individual tests, excepting a level for the final test in a test process. The final significance level depends on the succeeding command. In case of omittion, all levels must be given at the succeeding command.

SIG ...Significance pattern defined by a test process, where 1,0 and* mean significance, non-significance and ignorance, respectively. Examples are shown as below.

SIG(111) ...significance in view of a test process, when all individual tests are significant.

SIG $(11 *) \cdots$ significance in view of a test process, where the first and the second tests are significant. The third test does not work anything.

SIG $(1 * 0) \cdots$ significance in view of a test process, when the first test is significant, the third test is non-significant.

SIG(1) ...significance in view of a test process, specially when anyone of individual test is significant.

\section{(vi) Command TPATTN}

$\begin{aligned} \text { TPATTN } & \text { TEST (list of test name) } \\ & \text { POP (number of populations) } \\ & \text { SIZE (sample I sample } 2 \cdots) \\ & {[\text { TYPE (type of R.N.) }] } \\ & {[\text { ALPHA (probability level) }] } \\ & {[\text { SIM (size of simulation) }] } \\ & {[\text { PARA (parameters of R.N.) }] }\end{aligned}$

[Function]

To obtain frequencies for the respective significance and non-siginificance patterns due to several tests involved in a test process.

[Operands]

All operands are quite the same as those 
of POWER command.

(vii) Command TESTIM

TESTIM TEST (test name)

POP (number of populations)

SIZE (sample 1 sample $2 \cdots$ )

[TYPE (type of R.N.)]

[ALPHA (probability level)]

[SIM (size of simulation)]

[PARA (parameters of R.N.)]

[SIG (pattern of sig.)]

[Function]

To obtain the mean square error and value of a kind of risk function of estimating the mean of the first population after a preliminary test or a test process given.

[Operands]

TEST $\cdots$ The name of a test or a test process given. The name of tests involved in the process are shown in Table A, like those in POWER command.

ALPHA $\cdots$ A probability of significance level. In case of omittion, $\alpha=$ 0.05 .

The other operands are quite the same as those of POWER command.

\section{(viii) Command CONDIST}

CONDIST TEST (test process name)

POP (number of populations)

SIZE (sample 1 sample $2 \cdots$ )

[TYPE (type of R.N.)

[ALPHA (list of pro. level)]

[PAL (probability level) $]$

[SIM (size of simulation)]

[PARA (parameters of R. N.)] $[$ HIST]

\begin{abstract}
[Function]
To obtain the conditional distribution of test statistic used in the final test at a test-process (test series) defined.

[Operands]

TEST ...The name of a test-process defined by DEFTP command.

ALPHA ...Individual significance levels when they are not given by DEFTP command. See DEFTP command.

PAL …A significance level desired throughout the test process. Then a significant value of test statistic used in the final test at a test process is shown.
\end{abstract}

The other operands are the same as those used in POWER command.

Table A. Simple Statistical Tests Involved at Present*

\begin{tabular}{|c|c|c|}
\hline One-Sample Tests & Two-Sample Tests & Multi-Sample Tests \\
\hline $\begin{array}{l}\text { WiIcoxon one sample test } \\
\text { CHI-square test } \\
\text { KOLmogorov-Smirnov test }\end{array}$ & $\begin{array}{l}\text { Wilcoxon rank sum test } \\
\text { Ansari-Bradley test } \\
\text { LEPage test } \\
\text { CHI-square test } \\
\text { SIGN test } \\
\text { KOLmogorov-Smirnov test } \\
\text { D-test (Grove's) } \\
\text { Generalized Wilcoxon test } \\
\text { COX-Mantel test } \\
\text { MANtel-Haenzel test } \\
\text { T-test } \\
\text { WELch-aspin test } \\
\text { E-test }\end{array}$ & $\begin{array}{l}\text { KRUskal-wallis test } \\
\text { CHI-square test } \\
\text { FRIEdman test } \\
\text { MEDian test } \\
\text { LOGrank test }\end{array}$ \\
\hline
\end{tabular}


Table B. Types of Random Numbers and their Input-Forms

\begin{tabular}{|c|c|c|c|c|c|c|c|}
\hline \multirow{2}{*}{\multicolumn{2}{|c|}{ Types (notations) }} & \multicolumn{6}{|c|}{ Input-Forms } \\
\hline & & \multicolumn{2}{|c|}{ Ist Parameter } & \multicolumn{2}{|c|}{ 2nd Parameter } & \multicolumn{2}{|c|}{ 3rd Parameter } \\
\hline$\underline{\text { REctangular }}$ & {$[a, b]$} & $a$ & {$[0]$} & $b$ & {$[1]$} & & \\
\hline BInomial & $B(n, p)$ & $n$ & {$[10]$} & p & {$[0.5]$} & & \\
\hline POisson & mean a & $a$ & {$[5]$} & - & - & & \\
\hline NORmal & $N(a, b)$ & $a$ & {$[0]$} & b & {$[1]$} & & \\
\hline EXPonential & $b e^{-b(x-a)}$ & a & {$[0]$} & $b$ & {$[1]$} & & \\
\hline WE IbUIl & $\begin{array}{c}(c / b) Z^{c-1} e^{-Z^{c}} \\
Z \equiv(X-a) / b\end{array}$ & $a$ & [0] & $\mathrm{b}$ & [1] & c & [3] \\
\hline
\end{tabular}

[ ]: Value in case of omission for key-in.

\subsection{A simple illustration of the correspondence of command TESTIM with the mathematical formulation}

(i) Problem: The inference of population mean after one-sided $t$-test (Asano [6]).

Let $O_{n_{1}}:\left(x_{11}, x_{12}, \cdots, x_{1 n_{1}}\right)$ be a random sample of $n_{1}$ from a normal population $N\left(\mu_{1}, \sigma^{2}\right)$ and let $O_{n_{2}}:\left(x_{21}, x_{22}, \cdots, x_{2 n_{2}}\right)$ be another random sample of $n_{2}$ drawn from another normal population $N\left(\mu_{2}, \sigma^{2}\right)$. These two normal populations are known to have the same population variance $\sigma^{2}$, whose value is however unknown. Then, our rule of the statistical procedure is formulated in the following way.

(1) Let $\bar{x}_{i}$ and $s_{i}^{2}$ be the sample mean and the sample variance defined by $O_{n_{i}}$ $(i=1,2)$.

(2) Let the statistic $t$ be defined by

$$
t=\left(\vec{x}_{1}-\vec{x}_{2}\right) /\left\{\left(\frac{1}{n_{1}}+\frac{1}{n_{2}}\right)^{1 / 2}\left(\frac{n_{1} s_{1}^{2}+n_{2} s_{2}^{2}}{n_{1}+n_{2}-2}\right)^{1 / 2}\right\} .
$$

(3) Let us define the statistic $\overline{\bar{x}}$ in the following way:

(a) $\bar{x}=\bar{x}_{2}, \quad$ if $t \leqq \lambda$,

(b) $\overline{\bar{x}}=\left(n_{1} \bar{x}_{1}+n_{2} \bar{x}_{2}\right) /\left(n_{1}+n_{2}\right), \quad$ if $\lambda<t<A$,

(c) $\overline{\bar{x}}=\bar{x}_{1}$, if $t \geqq \Lambda$,

where the switching constants $\Lambda$ and $\lambda$ are prescribed constants.

THEOREM 4.1. The distribution function of $\overline{\bar{x}}$ is given by

$$
\begin{aligned}
\operatorname{Pr} .\{\overline{\bar{x}}<u\}= & \frac{1}{\sqrt{ } 2 \pi} \int_{T \leqq 2} \int e^{-p^{2} / 2} \frac{V^{\frac{n_{1}+n_{2}}{2}-2} e^{-V}}{\Gamma\left(\frac{n_{1}+n_{2}-2}{2}\right)} \Phi\left(\frac{u-\mu_{2}}{\sigma} \sqrt{n_{1}+\overline{n_{2}}}-\sqrt{\left.\frac{n_{1}}{n_{2}} p\right) d p d V}\right. \\
& +\frac{1}{\sqrt{ } 2 \bar{\pi}} \Phi\left(\frac{\left(n_{1}+n_{2}\right) u-\left(n_{1} \mu_{1}+n_{2} \mu_{2}\right)}{\sigma \sqrt{n_{1}+n_{2}}}\right) \int_{\lambda<T<1} \int e^{-\mu^{2} / 2} \frac{V^{\frac{n_{1}+n_{2}}{2}-2} e^{-V}}{\Gamma\left(\frac{n_{1}+n_{2}-2}{2}\right)} d p d V
\end{aligned}
$$




$$
+\frac{1}{\sqrt{ } 2 \pi} \int_{T \geq A} \int e^{-p^{2} / 2} \frac{V^{\frac{n_{1}+n_{2}}{2}-2} e^{-V}}{\Gamma\left(\frac{n_{1}+n_{2}-2}{2}\right)} \Phi\left(\frac{u-\mu_{1}}{\sigma} \sqrt{n_{1}+n_{2}}+\sqrt{\frac{n_{2}}{n_{1}}} p\right) d p d V,
$$

where

$$
T \equiv\left(\sqrt{\frac{n_{1} n_{2}}{n_{1}+n_{2}}} \frac{\mu_{2}-\mu_{1}}{\sigma}+p\right) / \sqrt{\frac{2}{n_{1}+n_{2}-2} V} .
$$

THEOREM 4.2. The mean value $E\{\overline{\bar{x}}\}$ and the mean square error M.S.E. $\{\overline{\bar{x}}\}$ of estimate $\overline{\bar{x}}$ are given by

$$
\begin{aligned}
\begin{aligned}
E\{\overline{\bar{x}}\}= & \mu_{1}-\frac{\left(\mu_{1}-\mu_{2}\right)}{n_{1}+n_{2}}\left\{n_{1} \operatorname{Pr} .\left\{D_{0}\right\}+n_{2}\left(1-\operatorname{Pr} .\left\{D_{2}\right\}\right)\right\} \\
+ & \frac{\sigma}{\sqrt{n_{1}+n_{2}}} \cdot\left\{\sqrt{\frac{n_{1}}{n_{2}}} I_{D_{0}}(p)-\sqrt{\frac{n_{2}}{n_{1}}} I_{D_{2}}(p)\right\}, \\
\text { M.S.E. }\{\overline{\bar{x}}\}= & \frac{\sigma^{2}}{n_{1}+n_{2}}+\frac{\sigma^{2}}{n_{1}+n_{2}}\left\{\frac{n_{2}}{n_{1}} I_{D_{2}}\left(p^{2}\right)+\frac{n_{1}}{n_{2}} I_{D_{0}}\left(p^{2}\right)\right\}+\left\{1-\operatorname{Pr} .\left\{D_{2}\right\}\right\} \\
& \cdot\left(\frac{n_{2}\left(\mu_{1}-\mu_{2}\right)}{n_{1}+n_{2}}\right)^{2}+\operatorname{Pr} .\left\{D_{0}\right\} \cdot\left\{\mu_{1}+\frac{n_{2} \mu_{1}+n_{1} \mu_{2}}{n_{1}+n_{2}}\right\}\left\{\mu_{2}+\frac{n_{1} \mu_{1}+n_{2} \mu_{2}}{n_{1}+n_{2}}\right\},
\end{aligned}
\end{aligned}
$$

where

$$
\begin{aligned}
& I_{D_{i}}\left(p^{j}\right)=\iint_{D_{i}} p^{j} f(p, V) d p d V, \quad(j=1,2), \\
& f(p, V)=\frac{1}{\sqrt{ } 2 \pi} e^{-p^{2} / 2} \frac{V^{\frac{n_{1}+n_{2}}{2}-2} e^{-V}}{\Gamma\left(\frac{n_{1}+n_{2}-2}{2}\right)}, \\
& D_{i}: \begin{cases}T \leqq \lambda, & (i=0), \\
T \geqq A, & (i=2),\end{cases} \\
& \operatorname{Pr} .\left\{D_{i}\right\}=\iint_{D_{i}} f(p, V) d p d V .
\end{aligned}
$$

(ii) Solution: For a practical situation, when the estimate of $\mu_{1}$ is given by a result of preliminary test of signficance between two population means, i. e. $\bar{x}=\bar{x}_{1}$ for the significance and $\bar{x}=\left(n_{1} \bar{x}_{1}+n_{2} \bar{x}_{2}\right) /\left(n_{1}+n_{2}\right)$ for the nonsignificance, where $\lambda=-\infty$ in the previous theorems, the properties of such a procedure are evaluated in the following way. 


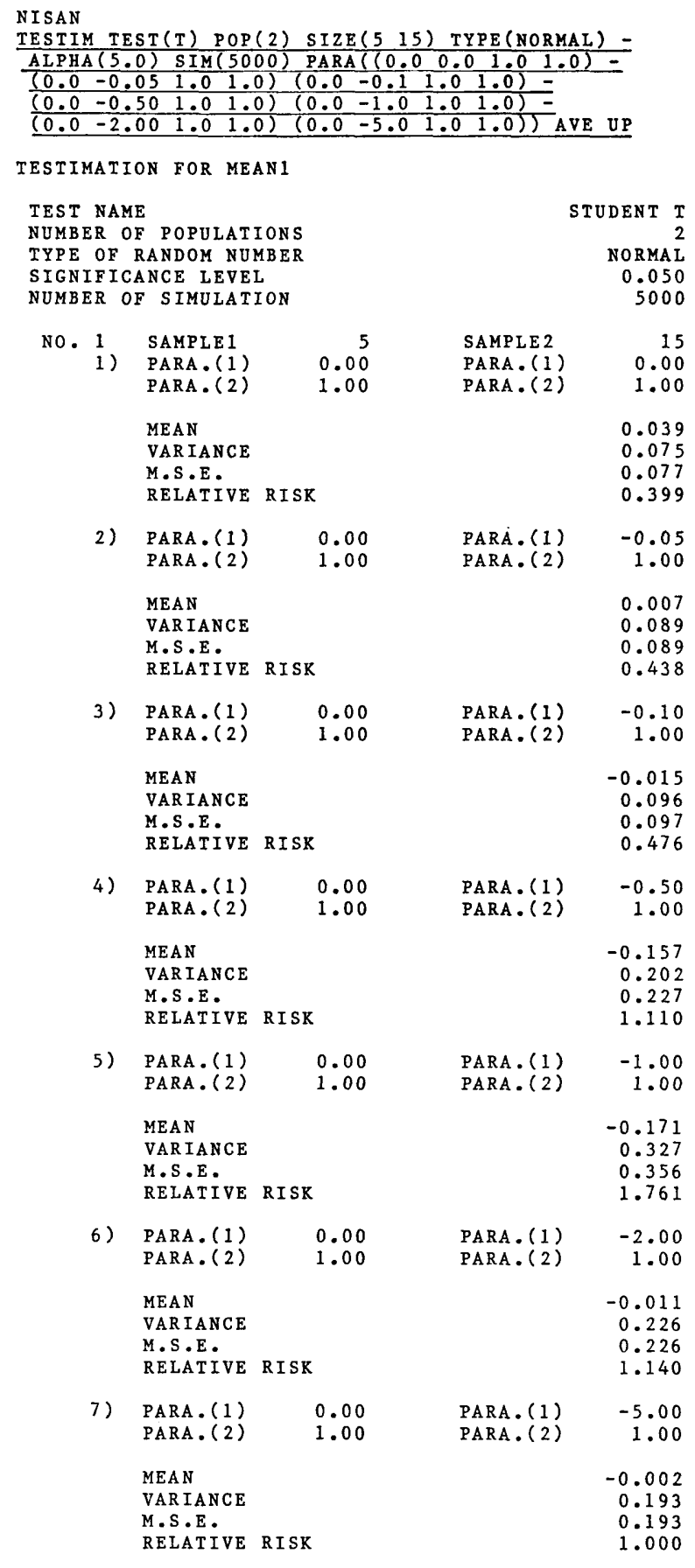




\subsection{The other illustrations of outputs}

(i) Command DIST (jointly checking the precision)

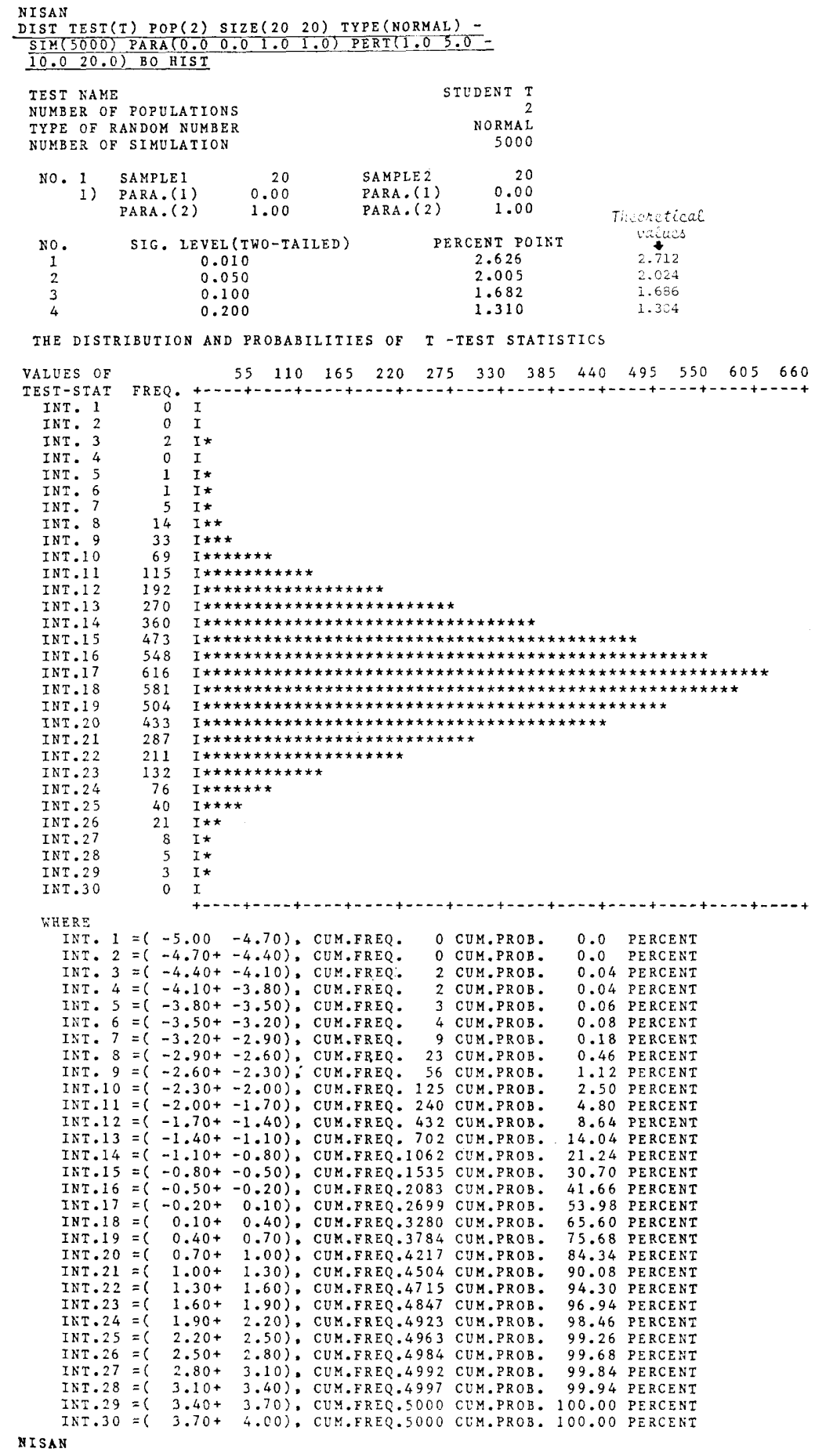


(ii) COMMAND POWER (jointly changing sampling ratio)

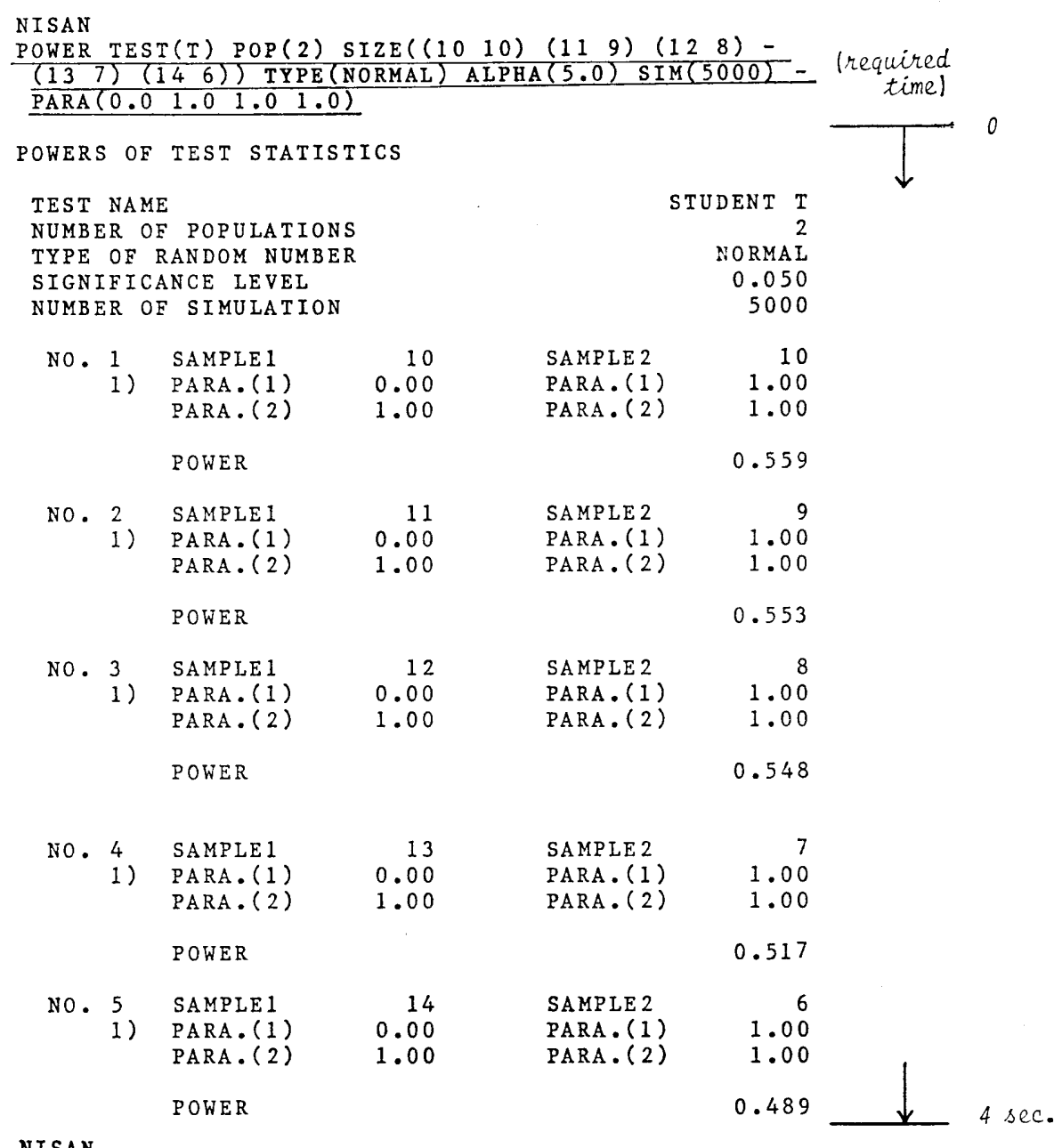


(iii) COMMAND POWER (for a process of testing)

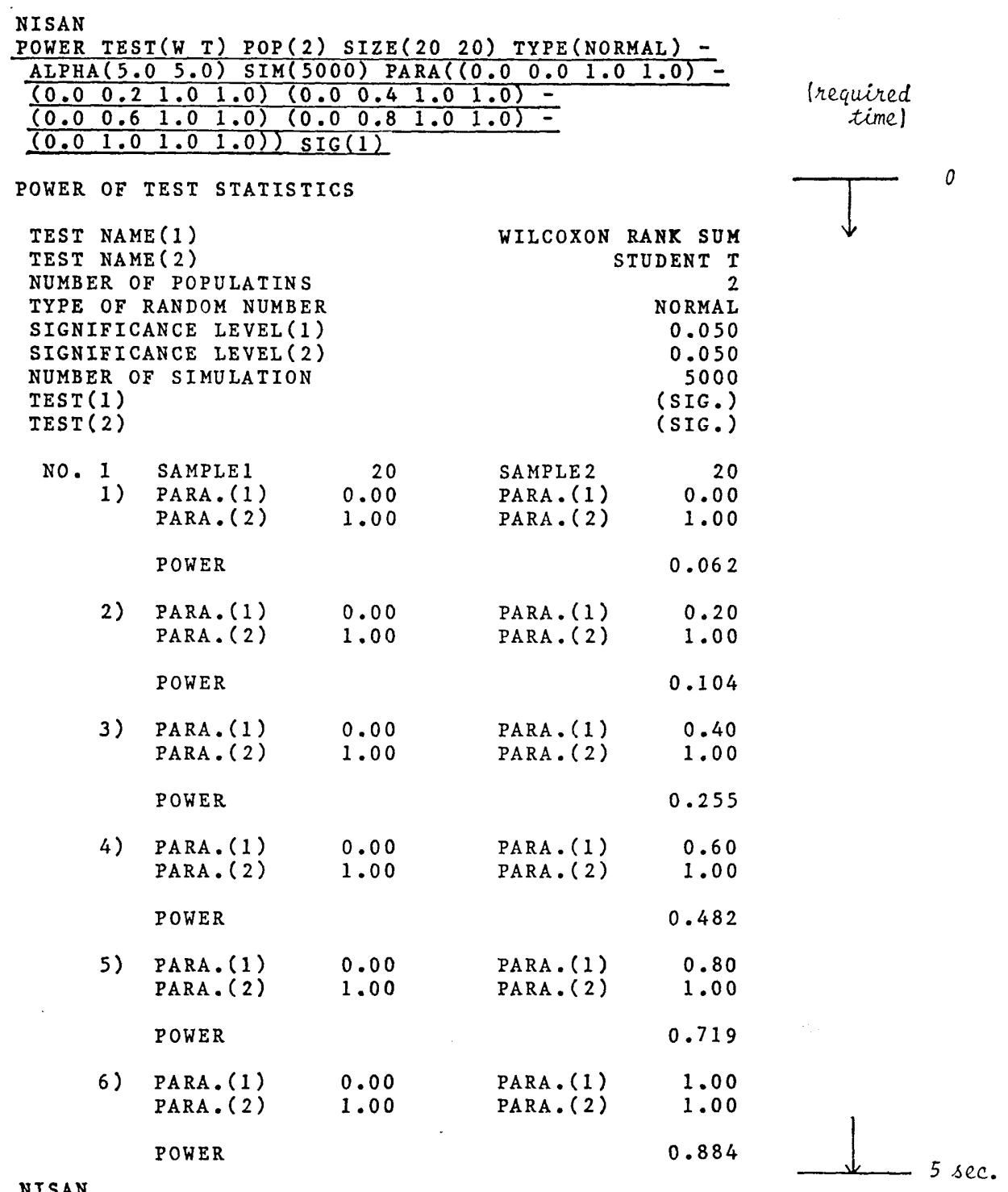


(iv) COMMAND TPATTN

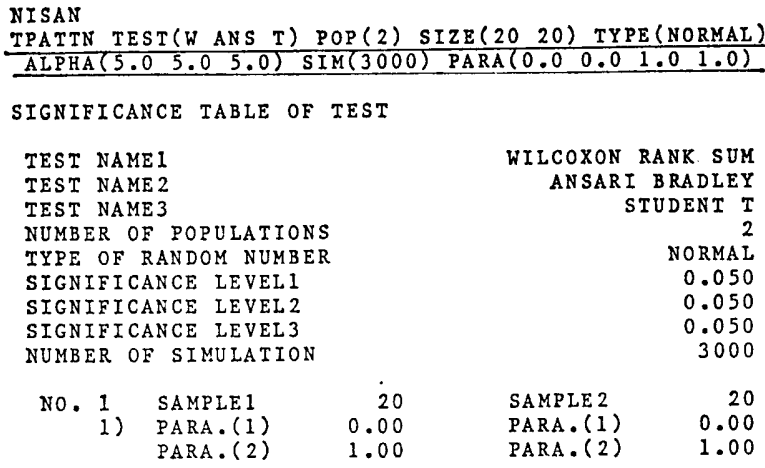

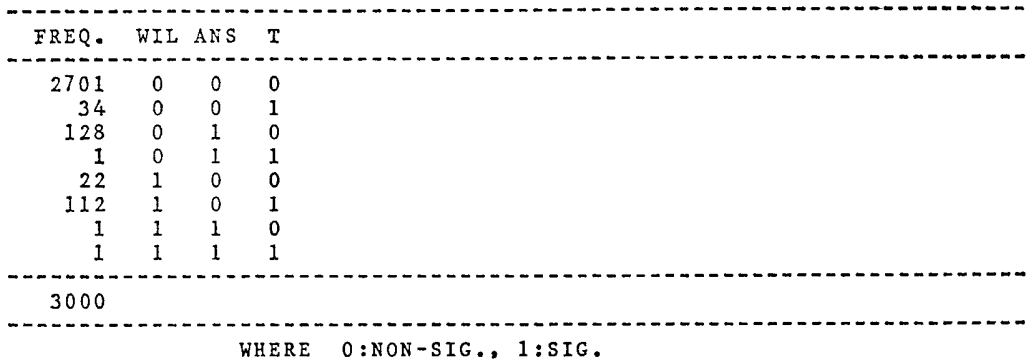

NISAN

(v) Command REF




(vi) Commands DEFTP And TESTIM

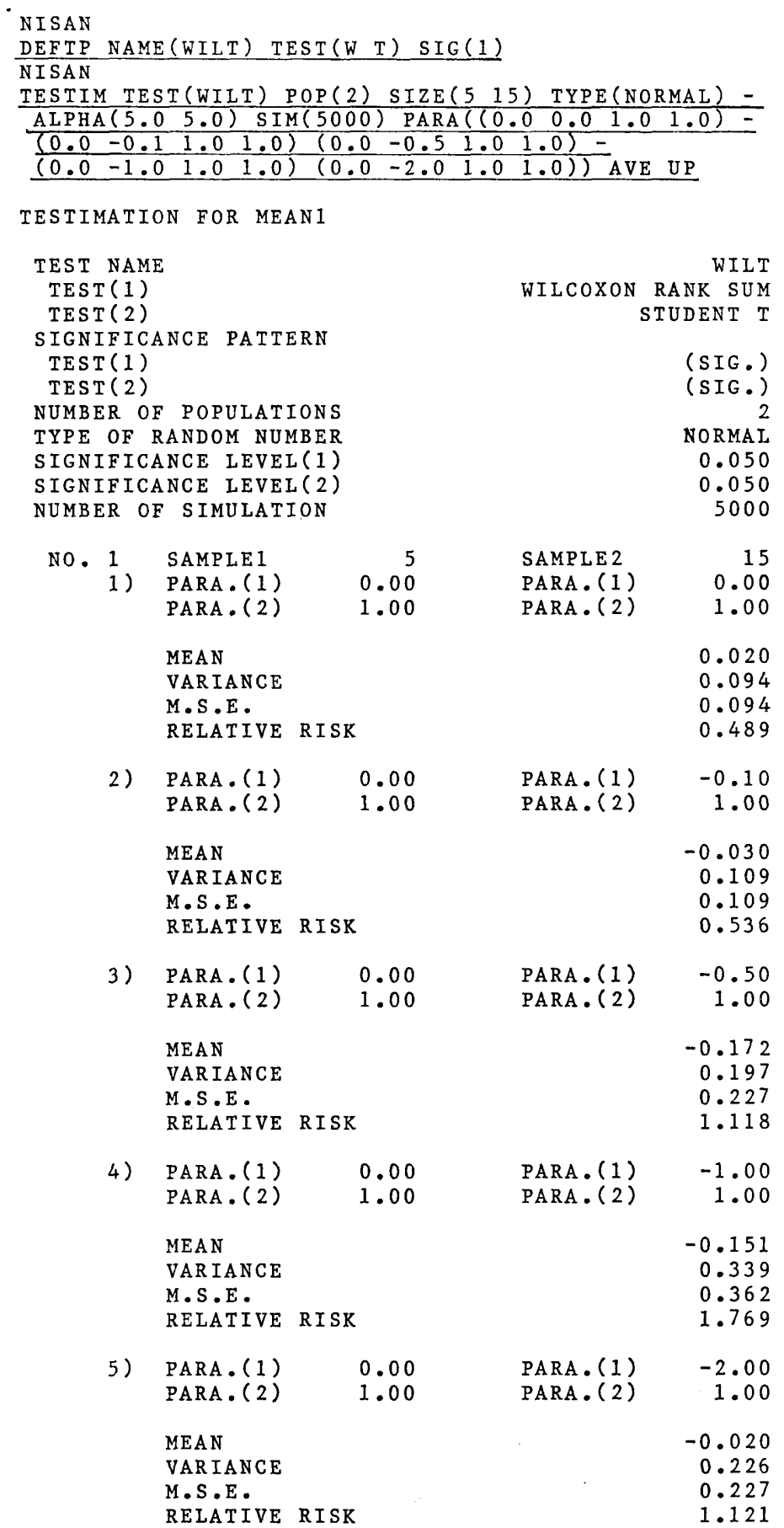




\section{(vii) Command CONDIST}

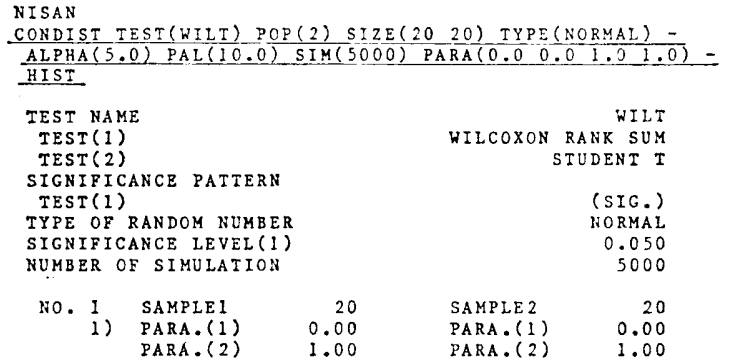

PERCENT POINT OF TEST(2) WITH TOTAL SIG. IEVEL FIXED

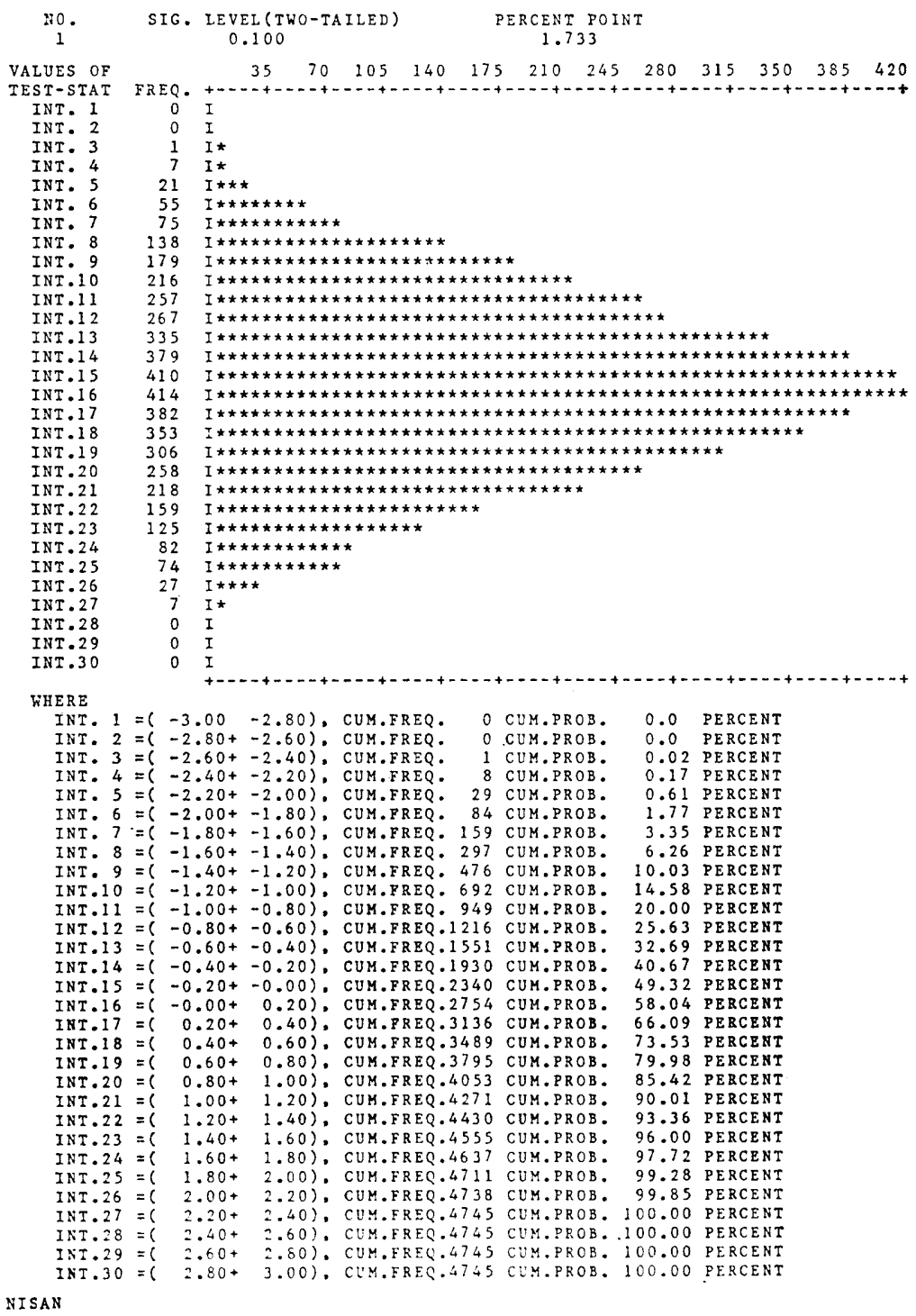




\section{Conclusion}

A new development of NISAN system is presented mainly in view of the functions of NISAN monitor, fully written by FORTRAN, and some of knowledge-commands newly-introduced, with ordinary method-commands sampled. The functions of monitor is for the benefit not only of the users, but also of the cooperation of NISAN research members. The functions of knowledge-commands are emphasized to be effective on statistical study and data analysis in practice for the senior statistician and the beginner, because of reasonable eveluation and preventing misapplication of statistical methods.

NISAN system is still developing into fullness on the ordinary usefulness and with the originality of statistical research.

\section{References}

[1] Asano, Сh., Wakimoto, K., Shohoj, T. et Al.: The statistical principle and methodo$\log y$ in NISAN system, Proceedings in Computational Statistics, Physica-Verlag (1978).

[2] Goto, M., Les.jk. H. AND Asano, $\mathrm{C}_{\mathrm{H} .:}$ : Methodology of data investigation in NISAN system; multivariate normality tests, Res. Rep. No. 90, Res. Instit. Fund. Inform. Sc., Kyushu University (1978).

[3] Asano, Ch., Tanaka, K., Jojima, K., Nishigori, N.: Command Construction Manual for NISAN Research Members, Res. Rep. No. 101, Res. Instit. Fund. Inform. Sc., Kyushu University, (1981).

[4] Asano, Ch., Shohoji, T., Wakimoto, K. ET Al.: System construction of new interactive statistical analy'sis (NISAN), Proceeding of Intern. Statist. Instit. (1979).

[5] Asano, Ch., Watanabe, M. and Hosokawa, K.: NISAN System Users' Guide, Res. Instit. Fund. Inform. Sc. (1981).

[6] Asaxo, CH.: Estimation after preliminary test of significance and their applications to biometrical researches, Bull. Math. Stat., 9, (1960), 1-23.

[7] KitagawA, T.: Successive process of statistical inferences (5), Mem. Fac. of Sc., Kyushu University, Ser. A, 7 (1953), 81-106.

[8] BANCROFT, T.A. AND HAN, C.P.: Inference based on conditional specification: A note and a bibliography., International Statistical Review, 45 (1977) 117-127. 